\title{
THE DISCRETE INTEGRAL MAXIMUM PRINCIPLE AND ITS APPLICATIONS
}

\author{
Thierry COUlHon, AleXANDER Grigor’yan AND FABio ZuCCA
}

(Received December 3, 2003)

Abstract. We prove an integral maximum principle for random walks on graphs, and give several applications to pointwise estimates of their transition probabilities, including the time-dependent case.

1. Introduction. Consider the heat equation $\partial_{t} u=\Delta u$, where $u=u(t, x)$ is a function on $\boldsymbol{R}_{+} \times \boldsymbol{R}^{n}$ and $\Delta$ is the Laplace operator in $\boldsymbol{R}^{n}$. It is well-known that, for all $p \in[1,+\infty]$, the $L^{p}$-norm of a solution $u(t, \cdot)$ is a non-increasing function of $t$. In the case $p=+\infty$, this statement is a particular case of the classical parabolic maximum principle. In the case $p<+\infty$, we will refer to it as an integral maximum principle. It admits the following generalization for weighted norms. Let a smooth function $\xi(t, x)$ defined on $\boldsymbol{R}_{+} \times \boldsymbol{R}^{n}$ be such that

$$
\partial_{t} \xi+\frac{1}{2}|\nabla \xi|^{2} \leq 0
$$

Then the weighted integral

$$
\int_{\boldsymbol{R}^{n}} u^{2}(t, x) e^{\xi(t, x)} d x
$$

is a non-increasing function of $t$. For example, the function $\xi(t, x)=d^{2}(x) / 2 t$ satisfies (1.1) provided $|\nabla d| \leq 1$.

The fact that the weighted integral (1.2) decreases in time remains true if the Laplace operator is replaced by a more general second-order elliptic operator in divergence form (under accordingly modified condition (1.1)). This was observed by Aronson and was used by him in [1] for obtaining two-sided estimates of the fundamental solutions of the corresponding heat equation. Similar results for the heat equation on a Riemannian manifold were obtained by the second author in [15], [14], [16]. Note that such results are universal, in the sense that they do not depend on the geometry on the manifold, and that they are instrumental in obtaining basic heat kernel estimates.

2000 Mathematics Subject Classification. Primary 60J15; Secondary 35B50, 39A12.

Key words and phrases. Discrete integral maximum principle, random walks on graphs, upper and lower estimates for a discrete heat kernel, time-dependent random walks, random walks on percolation clusters.

Research partially supported by the European Commission (IHP Network "Harmonic Analysis and Related Problems" 2002-2006, Contract HPRN-CT-2001-00273-HARP).

The third author was supported by a CNR Fellowship and by the University of Cergy-Pontoise. 
The purpose of this paper is to prove an analogue of the latter integral maximum principle in the setting of discrete heat equation on a graph. This will enable us to answer some basic questions about estimates of discrete heat kernels, which were left open in [9]. We should mention that the proof in the above continuous setting is quite easy, at least if $u$ and its derivatives decay fast enough as $x \rightarrow \infty$. Indeed, differentiating (1.2) in $t$ and applying integration by parts we obtain

$$
\begin{aligned}
\frac{d}{d t} \int_{\boldsymbol{R}^{n}} u^{2} e^{\xi} d x & =\int_{\boldsymbol{R}^{n}}\left(2 \Delta u u e^{\xi}+u^{2} \partial_{t} \xi e^{\xi}\right) d x \\
& =\int_{\boldsymbol{R}^{n}}\left(-2 \nabla u \cdot \nabla\left(u e^{\xi}\right)+u^{2} \partial_{t} \xi e^{\xi}\right) d x \\
& =\int_{\boldsymbol{R}^{n}}\left(-2|\nabla u|^{2} e^{\xi}-2 \nabla u \cdot \nabla \xi u e^{\xi}+u^{2} \partial_{t} \xi e^{\xi}\right) d x \\
& \leq \int_{\boldsymbol{R}^{n}}\left(-2|\nabla u|^{2}+\left(2|\nabla u|^{2}+\frac{1}{2}|\nabla \xi|^{2} u^{2}\right)+u^{2} \partial_{t} \xi\right) e^{\xi} d x \\
& =\int_{\boldsymbol{R}^{n}}\left(\frac{1}{2}|\nabla \xi|^{2}+\partial_{t} \xi\right) u^{2} e^{\xi} d x,
\end{aligned}
$$

which is non-positive by (1.1). However, if one tries to mimic this proof in the discrete setting, it does not work, due to additional terms that come from the discreteness of time. Before we can discuss this in details, let us introduce the necessary definitions and notation.

Let $\Gamma$ be a (non-oriented) countable graph, that is, a countable (we do not exclude the finite case) set of vertices, some of which are connected by edges. We write $x \sim y$ if $x$ and $y$ are connected by an edge (in this case we say that they are neighbors), and denote this edge by $\overline{x y}$. We shall assume throughout that $\Gamma$ is locally finite, that is, each $x \in \Gamma$ has a finite number of neighbors. A path of length $n$ between $x$ and $y$ in $\Gamma$ is a sequence $x_{i}, 0=1, \ldots, n$ such that $x_{0}=x, x_{n}=y$ and $x_{i} \sim x_{i+1}, i=0, \ldots, n-1$. We shall assume that $\Gamma$ is connected, i.e., there exists a path between any two points of $\Gamma$. Let $d$ be the graph metric on $\Gamma ; d(x, y)$ is the minimal length of a path between $x$ and $y$. Denote by $B(x, r)=\{y \in \Gamma ; d(x, y) \leq r\}$ the closed ball of radius $r \geq 0$ centered at $x \in \Gamma$.

Let $\mu_{x y}=\mu_{y x}$ be a non-negative symmetric weight defined for all $x, y \in \Gamma$ and vanishing on all pairs $x, y$ that are not neighbors (so that $\mu_{x y}$ is a function on the edge set). Assume $\mu$ is non-degenerate in the sense that for any $x \in \Gamma$ there exists $y \in \Gamma$ such that $\mu_{x y}>0$. The couple $(\Gamma, \mu)$ is called a weighted graph. Any graph $\Gamma$ admits the standard weight: $\mu_{x y}=1$ for all $x \sim y$.

A weight $\mu$ induces a positive weight $m$ on vertices defined by

$$
m(x)=\sum_{y \sim x} \mu_{x y},
$$

which extends to a measure on $\Gamma$ by

$$
|\Omega|=m(\Omega)=\sum_{x \in \Omega} m(x),
$$


for $\Omega \subset \Gamma$. Next, define a kernel $p(x, y)$ on $\Gamma \times \Gamma$ by

$$
p(x, y)=\frac{\mu_{x y}}{m(x)} .
$$

Note that $p$ is a Markov kernel, meaning that, for all $x, y \in \Gamma$,

$$
p(x, y) \geq 0 \quad \text { and } \quad \sum_{z \in \Gamma} p(x, z)=1,
$$

and $p$ is reversible with respect to measure $m$, that is,

$$
p(x, y) m(x)=p(y, x) m(y) .
$$

Conversely, given a Markov kernel $p$ reversible with respect to a positive measure $m$, the weight $\mu$ is uniquely determined by $\mu_{x y}=p(x, y) m(x)$.

Let $P$ be the Markov operator acting on functions on $\Gamma$ as follows

$$
P u(x):=\sum_{y \in \Gamma} p(x, y) u(y) \equiv \sum_{y \in \Gamma} h(x, y) u(y) m(y), \quad x \in \Gamma .
$$

The (discrete) Laplace operator $\Delta$ of $(\Gamma, \mu)$ is defined by $\Delta=P-\mathrm{Id}$, that is,

$$
\Delta u(x)=\sum_{y \in \Gamma} p(x, y)(u(y)-u(x)) .
$$

Let now $u=u(k, x)$ be a function on $N \times \Gamma$ where we regard the variable $k$ as a (discrete) time. It will be convenient to write $u_{k}=u(k, \cdot)$. Let $u$ satisfy the (discrete) heat equation, that is,

$$
u_{k+1}-u_{k}=\Delta u_{k}
$$

which is equivalent to $u_{k+1}=P u_{k}$. Let $f$ be a non-negative function on $N \times \Gamma$, which will play the role of a weight. Consider the following weighted $L^{2}$-norm of $u$ :

$$
I_{k}:=\sum_{x \in \Gamma} u_{k}^{2}(x) f_{k}(x) m(x) .
$$

Our main result, Theorem 2.2, says that $I_{k}$ is a non-increasing function of $k$ provided there exists $\alpha>0$ such that

$(\alpha)$

$$
p(x, x) \geq \alpha \quad \text { for all } x \in \Gamma
$$

and $f$ satisfies the inequality

$$
f_{k+1}-f_{k}+\frac{\left|\nabla f_{k+1}\right|^{2}}{4 \alpha f_{k+1}} \leq 0,
$$

where

$$
|\nabla f|^{2}(x):=\sum_{y \in \Gamma}(f(y)-f(x))^{2} p(x, y) .
$$


Note that in the continuous setting (1.1) implies that the function $f=e^{\xi}$ satisfies the inequality

$$
\partial_{t} f+\frac{|\nabla f|^{2}}{2 f} \leq 0,
$$

which matches (1.6).

Condition $(\alpha)$ has no analogue in the manifold setting. In the graph setting, it appears in [11] and [13]. At first sight it seems very restrictive; indeed, the simplest graph $Z^{D}$ with the standard weight $\mu$ does not satisfy it. However, for most applications of the integral maximum principle, it is possible to get rid of $(\alpha)$ by considering a new graph $\tilde{\Gamma}$, called the iterated graph, that has the same set of vertices as $\Gamma$ but $x$ and $y$ are related by an edge in $\tilde{\Gamma}$ if $d(x, y) \leq 2$ in $\Gamma$. Respectively, one considers on $\tilde{\Gamma}$ the Markov kernel $\tilde{p}(x, y)=$ $p_{2}(x, y)$, which is reversible with respect to the same measure $m(x)$. The associated weight is denoted by $\tilde{\mu}$. The weighted graph $(\tilde{\Gamma}, \tilde{\mu})$ satisfies Condition $(\alpha)$ provided $(\Gamma, \mu)$ satisfies the following condition

$$
m(B(x, 1))=\sum_{y \sim x} m(y) \leq \beta m(x), \quad x \in \Gamma
$$

for a constant $\beta$ (see Lemma 3.2). It is frequently possible to prove certain results about $p_{k}(x, y)$ on $\Gamma$ by having proved them first on $\tilde{\Gamma}$ for $\tilde{p}_{k}(x, y)$ using $(\alpha)$ and then transferring them back to $\Gamma$. This way of using $(\alpha)$ was introduced by Delmotte [13] and later was applied also in [9]. Note that the construction of iterated graphs may serve another purpose, namely, extend our results from Markov chains with range one to Markov chains with bounded range.

All our applications of the integral maximum principle relate to estimates of the heat kernel on $(\Gamma, \mu)$. Let $p_{k}(x, y), k \in N$, be the $k$-th iterate power of $p(x, y)$, that is,

$$
p_{0}(x, y)=\delta_{x, y}:= \begin{cases}0, & x \neq y, \\ 1, & x=y,\end{cases}
$$

and

$$
p_{k}(x, y)=\sum_{z \in \Gamma} p_{k-1}(x, z) p(z, y), \quad k \geq 1 .
$$

The function $p_{k}(x, y)$ is the $k$-th step transition function of the random walk defined by the transition probabilities $p(x, y)$. Define the transition density or the heat kernel of this random walk by

$$
h_{k}(x, y)=\frac{p_{k}(x, y)}{m(y)} .
$$

Note that unlike $p_{k}(x, y)$, the function $h_{k}(x, y)$ is symmetric in $x, y$. It follows from (1.7) that $h_{k}$ satisfies the identity

$$
h_{k+l}(x, y)=\sum_{z \in \Gamma} h_{k}(x, z) h_{l}(z, y) m(z),
$$

for any $k, l \in N$ and $x, y \in \Gamma$. Note that $x \mapsto h_{k}(x, y)$ is a solution of the discrete heat equation for any $y \in \Gamma$. For various aspects of heat kernel estimates on graphs, we refer the 
reader to the book [26], to the surveys [7], [10], [22] and to the references therein. Some of the lecture courses contained in [3] are also relevant.

Our purpose here is to provide with the integral maximum principle a basic and universal tool for the study of pointwise estimates of transition probabilities of random walks of graphs. As such, it does not use specific geometric properties of the graph, such as the volume growth or the Poincaré inequalities. On the other hand, it is very stable, so that it might prove useful for instance in the study of random walks in random environment.

In Section 2 we prove the integral maximum principle for graphs satisfying Condition $(\alpha)$. In Section 3 we discuss the relation between Conditions $(\alpha)$ and $(\beta)$. In subsequent sections, we present a selection of three applications of the integral maximum principle for graphs satisfying $(\alpha)$ or $(\beta)$.

Corollary 4.2 says that, for all finite subsets $A$ and $B$ in $\Gamma$,

$$
\sum_{x \in A} \sum_{y \in B} h_{k}(x, y) m(x) m(y) \leq C \exp \left(-c \frac{d^{2}(A, B)}{k}\right) \sqrt{m(A) m(B)},
$$

where $d(A, B):=\inf \{d(x, y) ; x \in A, y \in B\}$ is the distance between $A$ and $B$, and $C, c$ are positive constants. The inequality (1.9) is not new. An analogue of (1.9) for heat kernels on Riemannian manifolds was proved by Davies [12, Theorem 2] (see also [19] for an earlier version and [17] for alternative proofs). In the graph case, when $A$ and $B$ are single points, the inequality (1.9) yields

$$
p_{k}(x, y) \leq C \exp \sqrt{\frac{m(y)}{m(x)}}\left(-c \frac{d^{2}(x, y)}{k}\right) .
$$

A weaker version of this estimate is due to Varopoulos [24], and the proof in full generality is due to Carne [5]. Moreover, Carne's method allows to prove (1.9) for arbitrary sets $A, B$ without assuming $(\alpha)$ or $(\beta)$. Another proof of (1.9) was obtained in [9, Lemma 5.1] using a result of Hebisch and Saloff-Coste [18] for an auxiliary random walk with continuous time. By the way, in the statement of the Davies-Gaffney inequality in [9, Lemma 5.1], one hypothesis is missing. Namely, one has to assume that

$$
\sup _{x, y \in \Gamma} \frac{\mu_{x y}}{m(x) m(y)}<\infty,
$$

in order to be able to apply [18, Lemma 2.4]. In Section 4, we deduce (1.9) from the integral maximum principle to illustrate the strength of the latter. We also deduce another, apparently new, generalization to arbitrary sets of the Carne-Varopoulos estimate, namely,

$$
\sum_{x \in A} \sum_{y \in B} h_{k}^{2}(x, y) m(x) m(y) \leq C \exp \left(-c \frac{d^{2}(A, B)}{k}\right) \min (\operatorname{card}(A), \operatorname{card}(B)) .
$$

Another application of the integral maximum principle enables one to obtain off-diagonal estimates of the heat kernel from on-diagonal ones. Assume that for two fixed points $x, y \in \Gamma$ 
and all $k \in N$ the following estimates hold:

$$
h_{2 k}(x, x) \leq \frac{1}{f(k)} \quad \text { and } \quad h_{2 k}(y, y) \leq \frac{1}{g(k)},
$$

where $f$ and $g$ are some increasing regular enough functions. Then, for all $k \in N$,

$$
h_{2 k}(x, y) \leq \frac{C}{\sqrt{f(\eta k) g(\eta k)}} \exp \left(-c \frac{d^{2}(x, y)}{k}\right),
$$

for some positive constants $C, c, \eta$ (see Theorem 5.2). An analogous result for manifolds was proved in [16]. Let us emphasize that unlike other methods for obtaining Gaussian upper bounds (see for example [9] and [18]), we need information on the heat kernel only at fixed points $x, y$, which provides a lot of flexibility for potential applications.

The integral maximum principle also enables one to obtain a lower bound of heat kernel from an upper bound, similarly to a result of [8, Theorem 7.2] for the manifolds setting. Assume that for a fixed point $x \in \Gamma$ the following two conditions hold:

$$
V(x, 2 r) \leq C V(x, r) \text { for all } r>0,
$$

where $V(x, r):=m(B(x, r))$, and

$$
h_{k}(x, x) \leq \frac{C}{V(x, \sqrt{k})}, \quad \text { for all } k \in N .
$$

Then, by Theorem 6.1, there exists a constant $c>0$ such that

$$
h_{k}(x, x) \geq \frac{c}{V(x, \sqrt{k})} \quad \text { for all } k \in N .
$$

In Section 7 we observe that our results can be carried over to time-dependent random walks, and in Section 8 we give an application of Theorem 5.2 to random walks on percolation clusters.

We thank Pierre Mathieu for discussions on random walks on percolation clusters.

2. The discrete integral maximum principle. We start with the following simple observation, which will not be used, but which gives some flavor of what follows.

Proposition 2.1. Let $(\Gamma, \mu)$ be a weighted graph and let $u$ be a solution of the discrete heat equation on $(\Gamma, \mu)$. Let $f$ be a non-negative function on $\mathbf{N} \times \Gamma$ such that

$$
P f_{k+1} \leq f_{k} \text { for all } k \in N .
$$

Then, for any $q \in[1,+\infty)$, the quantity

$$
J_{k}^{(q)}:=\sum_{x \in \Gamma}\left|u_{k}(x)\right|^{q} f_{k}(x) m(x)
$$

is non-increasing in $k$, that is, $J_{k+1}^{(q)} \leq J_{k}^{(q)}$ for all $k \in N$.

Proof. Since

$$
u_{k+1}(x)=\left(P u_{k}\right)(x)=\sum_{y \in \Gamma} p(x, y) u_{k}(y)
$$


we obtain, using the Hölder inequality, (1.3), and (1.4),

$$
\begin{aligned}
J_{k+1}^{(q)} & =\sum_{x \in \Gamma}\left|\sum_{y \in \Gamma} p(x, y) u_{k}(y)\right|^{q} f_{k+1}(x) m(x) \\
& \leq \sum_{x \in \Gamma}\left(\sum_{y \in \Gamma} p(x, y)\right)^{q-1}\left(\sum_{y \in \Gamma} p(x, y)\left|u_{k}(y)\right|^{q}\right) f_{k+1}(x) m(x) \\
& =\sum_{y \in \Gamma} \sum_{x \in \Gamma} p(y, x)\left|u_{k}(y)\right|^{q} f_{k+1}(x) m(y) \\
& =\sum_{y \in \Gamma}\left|u_{k}(y)\right|^{q}\left(P f_{k+1}\right)(y) m(y) \\
& \leq \sum_{y \in \Gamma}\left|u_{k}(y)\right|^{q} f_{k}(y) m(y)=J_{k}^{(q)} .
\end{aligned}
$$

As a simple consequence, by taking $f_{k} \equiv 1$, we see that the $l^{q}(\Gamma, m)$-norm of a solution of the discrete heat equation is non-increasing. This is of course also true if $q=+\infty$. From now on, we will consider only the case $q=2$.

Let us introduce the following notation: given a function $f$ on $N$ we write

$$
\partial_{k} f:=f_{k+1}-f_{k} .
$$

It is easy to see that

$$
\begin{aligned}
\partial_{k}(f g) & =\left(\partial_{k} f\right) g_{k+1}+f_{k}\left(\partial_{k} g\right) \\
\partial_{k}\left(f^{2}\right) & =2 f_{k} \partial_{k} f+\left(\partial_{k} f\right)^{2} .
\end{aligned}
$$

Similarly, if $f$ is a function on $\Gamma$ and $x, y$ are two vertices in $\Gamma$, let us set

$$
\nabla_{x y} f:=f(y)-f(x)
$$

and observe that the following product rules take place:

$$
\begin{aligned}
& \nabla_{x y}(f g)=f(x)\left(\nabla_{x y} g\right)+\left(\nabla_{x y} f\right) g(y) \\
& \nabla_{x y}\left(f^{2}\right)=2 f(x) \nabla_{x y} f+\left(\nabla_{x y} f\right)^{2} .
\end{aligned}
$$

Let us define $|\nabla f|$ as a function on $\Gamma$ by

$$
|\nabla f|^{2}(x)=\sum_{y \in \Gamma}\left(\nabla_{x y} f\right)^{2} p(x, y) .
$$

Note that the Laplace operator on $(\Gamma, \mu)$ can be rewritten in the form

$$
\Delta f(x)=\sum_{y \in \Gamma} p(x, y) \nabla_{x y} f .
$$

One can easily check the following integration by parts formula: if one of the functions $f, g$ on $\Gamma$ has a finite support then

$$
\sum_{x \in \Gamma} \Delta f(x) g(x) m(x)=-\frac{1}{2} \sum_{x, y \in \Gamma}\left(\nabla_{x y} f\right)\left(\nabla_{x y} g\right) \mu_{x y}
$$


(the factor $1 / 2$ appears because each edge is counted twice in the sum).

Given $a \in N$ and $b \in N \cup\{+\infty\}, a<b$, define the intervals

$$
[a, b)=\{k \in N ; a \leq k<b\} \quad \text { and } \quad[a, b]=\{k \in N ; a \leq k \leq b\} .
$$

Let $n \in N \cup\{+\infty\}$. We say that a function $u$ satisfies the heat equation in $[0, n) \times \Gamma$ if $u$ is defined in $[0, n] \times \Gamma$ and

$$
\partial_{k} u=\Delta u_{k} \quad \text { for all } k \in[0, n) .
$$

The next theorem is the main result of this section.

THEOREM 2.2. Let $(\Gamma, \mu)$ be a weighted graph satisfying Condition $(\alpha)$ and let $f$ be a strictly positive function on $[0, n] \times \Gamma$ such that, for all $x \in \Gamma$ and $k \in[0, n)$,

$$
\partial_{k} f(x)+\frac{\left|\nabla f_{k+1}\right|^{2}}{4 \alpha f_{k+1}}(x) \leq 0 .
$$

Then, for any solution $u$ of the heat equation in $[0, n) \times \Gamma$, the quantity

$$
J_{k}=J_{k}(u):=\sum_{x \in \Gamma} u_{k}^{2}(x) f_{k}(x) m(x)
$$

is non-increasing in $k$, that is, $J_{k+1} \leq J_{k}$ for any $k \in[0, n)$.

Proof. Assume first that $\operatorname{supp}\left(u_{0}\right)$ is a finite set, which implies that $\operatorname{supp}\left(u_{k}\right)$ is also finite for any $k \in[0, n)$ and which will ensure finiteness of all the sums in the argument below. By (2.1), we have

$$
\partial_{k}\left(u^{2} f\right)=\partial_{k}\left(u^{2}\right) f_{k+1}+u_{k}^{2} \partial_{k} f=2 u_{k}\left(\partial_{k} u\right) f_{k+1}+\left(\partial_{k} u\right)^{2} f_{k+1}+u_{k}^{2} \partial_{k} f,
$$

whence

$$
\begin{aligned}
\partial_{k} J(u)= & \sum_{x \in \Gamma} \partial_{k}\left(u^{2} f\right)(x) m(x) \\
= & 2 \sum_{x \in \Gamma} u_{k}(x) \partial_{k} u(x) f_{k+1}(x) m(x)+\sum_{x \in \Gamma}\left(\partial_{k} u(x)\right)^{2} f_{k+1}(x) m(x) \\
& +\sum_{x \in \Gamma} u_{k}^{2}(x) \partial_{k} f(x) m(x) .
\end{aligned}
$$

Using (2.5), (2.4), and (2.2) let us observe that the first sum in (2.7) is equal to

$$
\begin{aligned}
2 \sum_{x \in \Gamma} u_{k}(x) \Delta u_{k}(x) f_{k+1}(x) m(x) & \\
& =-\sum_{x, y \in \Gamma}\left(\nabla_{x y} u_{k}\right) \nabla_{x y}\left(u_{k} f_{k+1}\right) \mu_{x y} \\
& =-\sum_{x, y \in \Gamma}\left(\nabla_{x y} u_{k}\right)^{2} f_{k+1}(x) \mu_{x y}-\sum_{x, y \in \Gamma}\left(\nabla_{x y} u_{k}\right) u_{k}(y)\left(\nabla_{x y} f_{k+1}\right) \mu_{x y} \\
& =-\sum_{x, y \in \Gamma}\left(\nabla_{x y} u_{k}\right)^{2} f_{k+1}(x) \mu_{x y}-\sum_{x, y \in \Gamma}\left(\nabla_{x y} u_{k}\right) u_{k}(x)\left(\nabla_{x y} f_{k+1}\right) \mu_{x y},
\end{aligned}
$$


where in the last sum in (2.8), in order to replace $u_{k}(y)$ by $u_{k}(x)$, one switches $x$ and $y$ in the notation using $\nabla_{x y}=-\nabla_{y x}$ and $\mu_{x y}=\mu_{y x}$.

To handle the second term in (2.7), we will argue as in $[13, \S 1.5]$ and $[9$, Lemma 4.6] using Condition $(\alpha)$. Indeed, we have

$$
\begin{aligned}
\left(\partial_{k} u(x)\right)^{2} & =\left(\Delta u_{k}(x)\right)^{2} \\
& =\left(\sum_{y \in \Gamma \backslash\{x\}}\left(\nabla_{x y} u_{k}\right) p(x, y)\right)^{2} \\
& \leq\left(\sum_{y \in \Gamma \backslash\{x\}} p(x, y)\right)\left(\sum_{y \in \Gamma}\left(\nabla_{x y} u_{k}\right)^{2} p(x, y)\right) \\
& =(1-p(x, x)) \sum_{y \in \Gamma}\left(\nabla_{x y} u_{k}\right)^{2} p(x, y) \\
& \leq(1-\alpha) \sum_{y \in \Gamma}\left(\nabla_{x y} u_{k}\right)^{2} p(x, y),
\end{aligned}
$$

whence, using $p(x, y) m(x)=\mu_{x y}$,

$$
\sum_{x \in \Gamma}\left(\partial_{k} u(x)\right)^{2} f_{k+1}(x) m(x) \leq(1-\alpha) \sum_{x, y \in \Gamma}\left(\nabla_{x y} u_{k}\right)^{2} f_{k+1}(x) \mu_{x y} .
$$

Hence, substituting (2.8) and (2.10) into (2.7) and using (2.3) we obtain

$$
\begin{aligned}
\partial_{k} J(u) \leq-\alpha & \sum_{x, y \in \Gamma}\left(\nabla_{x y} u_{k}\right)^{2} f_{k+1}(x) \mu_{x y}-\sum_{x, y \in \Gamma}\left(\nabla_{x y} u_{k}\right) u_{k}(x)\left(\nabla_{x y} f_{k+1}\right) \mu_{x y} \\
& +\sum_{x \in \Gamma} u_{k}^{2}(x) \partial_{k} f(x) m(x) \\
=- & \sum_{x, y \in \Gamma}\left(\nabla_{x y} u_{k} \sqrt{\alpha f_{k+1}(x)}+\frac{u_{k}(x)}{2 \sqrt{\alpha f_{k+1}(x)}} \nabla_{x y} f_{k+1}\right)^{2} \mu_{x y} \\
& +\sum_{x \in \Gamma} u_{k}^{2}(x)\left(\frac{1}{4 \alpha f_{k+1}(x)}\left|\nabla f_{k+1}\right|^{2}(x)+\partial_{k} f(x)\right) m(x) .
\end{aligned}
$$

By the hypothesis (2.6), the expression in the brackets in the last sum is non-positive, whence $\partial_{k} J \leq 0$.

Let now $u_{0}$ be an arbitrary function on $\Gamma$. Without loss of generality, we can assume that $J_{0}(u)<\infty$. Indeed, as long as $J_{k}(u)=+\infty$ there is nothing to prove, and if $k_{0}$ is the minimum integer such that $J_{k_{o}}(u)<+\infty$, then we can shift the time as follows: $k \mapsto k-k_{0}$.

Let us take an increasing sequence of finite subsets $\left\{\Gamma_{i}\right\}_{i \in N}$ such that $\bigcup_{i=0}^{+\infty} \Gamma_{i}=\Gamma$ and define the initial states $u_{0}^{(i)}:=\mathbf{1}_{\Gamma_{i}} u_{0}$. Alongside the function $u_{k}=P^{k} u_{0}$ consider also the following functions:

$$
u_{k}^{(i)}:=P^{k} u_{0}^{(i)}, \quad v_{k}^{(i)}:=P^{k}\left|u_{0}^{(i)}\right|, \quad v_{k}:=P^{k}\left|u_{0}\right| .
$$


When $i \rightarrow \infty$ we have, for any $x \in \Gamma$ and $k \in N$,

$$
u_{k}^{(i)}(x) \rightarrow u_{k}(x) \quad \text { and } \quad v_{k}^{(i)}(x) \uparrow v_{k}(x) .
$$

By the monotone convergence theorem, we conclude

$$
J_{k}\left(v^{(i)}\right) \uparrow J_{k}(v) .
$$

Since $v^{(i)}$ is a solution with finite support, $J_{k}\left(v^{(i)}\right)$ is monotone decreasing in $k$ whence we see that so is $J_{k}(v)$. In particular, we have

$$
J_{k}(v) \leq J_{0}(v)=J_{0}(u)<\infty .
$$

Since $\left|u_{k}^{(i)}\right| \leq v_{k}^{(i)} \leq v_{k}$, we obtain by the dominated convergence theorem

$$
J_{k}\left(u^{(i)}\right) \rightarrow J_{k}(u) .
$$

Since $J_{k}\left(u^{(i)}\right)$ is monotone decreasing in $k$, we conclude that so is $J_{k}(u)$, which completes the proof.

In the next statement we shall give a first example of a non-trivial weight $f$ satisfying (2.6). This weight will be used in Section 4. We say that a function $\rho$ on $\Gamma$ is 1-Lipschitz if $\left|\nabla_{x y} \rho\right| \leq 1$ whenever $x \sim y$. For example, if $M$ is any subset of $\Gamma$ and $\rho(x)$ is a distance to $M$, that is, $\rho(x):=d(x, M)$, then $\rho$ is 1-Lipschitz.

Proposition 2.3. Let $(\Gamma, \mu)$ be a weighted graph satisfying Condition $(\alpha)$ and let $\rho(x)$ be a 1-Lipschitz function on $\Gamma$. Let $a$ and $b$ be two real numbers satisfying

$$
b \geq \log \left(1+\frac{\left(e^{|a|}-1\right)^{2}}{4 \alpha}\right) .
$$

Then, for any solution $u$ of the heat equation in $[0, n) \times \Gamma$, the quantity

$$
J_{k}=J_{k}(u):=\sum_{x \in \Gamma} u_{k}^{2}(x) e^{a \rho(x)-b k} m(x)
$$

is non-increasing in $k \in[0, n)$.

Proof. By Theorem 2.2, it suffices to prove that the function $f_{k}(x):=e^{a \rho(x)-b k}$ satisfies (2.6). We have

$$
\partial_{k} f(x)=\left(e^{-b}-1\right) f_{k}(x),
$$

and, for all $x, y \in \Gamma$ such that $x \sim y$,

$$
\left|\nabla_{x y} f_{k+1}(x)\right| \leq\left(e^{|a|}-1\right) f_{k+1}(x) .
$$

Therefore

$$
\left|\nabla f_{k+1}\right|^{2}(x)=\sum_{\{y ; y \sim x\}}\left|\nabla_{x y} f_{k+1}(x)\right|^{2} p(x, y) \leq\left(e^{|a|}-1\right)^{2} f_{k+1}^{2}(x),
$$

and

$$
\frac{\left|\nabla f_{k+1}(x)\right|^{2}}{f_{k+1}(x)} \leq\left(e^{|a|}-1\right)^{2} f_{k+1}(x)=e^{-b}\left(e^{|a|}-1\right)^{2} f_{k}(x) .
$$


Finally,

$$
\partial_{k} f(x)+\frac{\left|\nabla f_{k+1}(x)\right|^{2}}{4 \alpha f_{k+1}(x)} \leq\left[e^{-b}\left(1+\frac{\left(e^{|a|}-1\right)^{2}}{4 \alpha}\right)-1\right] f_{k}(x),
$$

and (2.6) follows from (2.11).

REMARK 2.4. Observe that there is a positive constant $c(\alpha)$ such that, for all $a \in \boldsymbol{R}$,

$$
\log \left(1+\frac{\left(e^{|a|}-1\right)^{2}}{4 \alpha}\right) \leq c(\alpha) a^{2} .
$$

Hence, (2.11) is satisfied by any couple $a, b$ with $b=c(\alpha) a^{2}$. The relation $b=c a^{2}$ between $a$ and $b$ is important in applications of Theorem 2.3; in those applications, one chooses $a$ to be a small positive number. Without Condition $(\alpha)$ one cannot ensure the existence of such a constant $c$ that the quantity (2.12) decays for any couple $a, b$ related by $b=c a^{2}$.

Another family of weight functions satisfying (2.6) is given by the following proposition. This weight will be used in Sections 5 and 6.

Proposition 2.5. Let $(\Gamma, \mu)$ be a weighted graph satisfying Condition $(\alpha)$ and let $\rho$ be a 1-Lipschitz function on $\Gamma$ such that inf $\rho \geq 1$. There exists a positive number $D_{\alpha}$ such that, for any $D \geq D_{\alpha}$, the weight function

$$
f_{k}(x)=f_{k}^{D}(x):=\exp \left(-\frac{\rho^{2}(x)}{D(n+1-k)}\right)
$$

satisfies (2.6) for all $x \in \Gamma$ and $k \in[0, n)$. Hence, for any solution $u$ of the heat equation in $[0, n) \times \Gamma$, the quantity $J_{k}:=\sum_{x \in \Gamma} u_{k}^{2}(x) f_{k}(x) m(x)$ is non-increasing in $k \in[0, n)$.

Proof. A simple calculation shows that

$$
\begin{aligned}
-\partial_{k} f(x) & =\left(\exp \left(\frac{\rho^{2}(x)}{D(n+1-k)(n-k)}\right)-1\right) f_{k+1}(x) \\
& \geq\left(\exp \left(\frac{\rho^{2}(x)}{2 D(n-k)^{2}}\right)-1\right) f_{k+1}(x)
\end{aligned}
$$

and

$$
\begin{aligned}
\left|\nabla f_{k+1}(x)\right|^{2} & =\sum_{y: y \sim x} p(x, y)\left(\exp \left(-\frac{\rho^{2}(y)}{D(n-k)}\right)-\exp \left(-\frac{\rho^{2}(x)}{D(n-k)}\right)\right)^{2} \\
& =f_{k+1}^{2}(x) \sum_{y: y \sim x} p(x, y)\left(\exp \left(\frac{\rho^{2}(x)-\rho^{2}(y)}{D(n-k)}\right)-1\right)^{2} .
\end{aligned}
$$

By the Lipschitz condition and the hypothesis $\rho(x) \geq 1$ we obtain

$$
\left|\rho^{2}(x)-\rho^{2}(y)\right|=|\rho(x)-\rho(y)||\rho(x)+\rho(y)| \leq 2 \rho(x)+1 \leq 3 \rho(x) .
$$

Next we use the following elementary inequality: if $|t| \leq s$ then

$$
\left|e^{t}-1\right| \leq e^{s}-1 .
$$


Combining together the previous lines, we obtain

$$
\left|\nabla f_{k+1}(x)\right|^{2} \leq f_{k+1}^{2}(x)\left(\exp \left(\frac{3 \rho(x)}{D(n-k)}\right)-1\right)^{2} .
$$

Next let us use another elementary fact: for any $A>0$ there exists $B>0$ such that, for all $t>0$,

$$
\left(e^{t}-1\right)^{2} \leq A e^{B t^{2}}-1 .
$$

Setting $t=3 \rho(x) / D(n-k)$ and $A=4 \alpha$ we obtain that, for some $B=B(\alpha)$,

$$
\frac{1}{4 \alpha}\left(\exp \left(\frac{3 \rho(x)}{D(n-k)}\right)-1\right)^{2} \leq \exp \left(\frac{B \rho^{2}(x)}{D^{2}(n-k)^{2}}\right)-1 .
$$

Hence, if $D \geq D_{\alpha}:=2 B$, then the right hand side of (2.16) is bounded from above by

$$
\exp \left(\frac{\rho^{2}(x)}{2 D(n-k)^{2}}\right)-1 \text {. }
$$

Combining with (2.14) and (2.15), we obtain

$$
\begin{aligned}
\frac{\left|\nabla f_{k+1}(x)\right|^{2}}{4 \alpha f_{k+1}(x)} & \leq \frac{f_{k+1}(x)}{4 \alpha}\left(\exp \left(\frac{3 \rho(x)}{D(n-k)}\right)-1\right)^{2} \\
& \leq f_{k+1}(x)\left(\exp \left(\frac{\rho^{2}(x)}{2 D(n-k)^{2}}\right)-1\right) \leq-\partial_{k} f(x),
\end{aligned}
$$

which was to be proved.

3. Iterated graph. Recall that with any weighted graph $(\Gamma, \mu)$ there associates an iterated graph $\tilde{\Gamma}$ whose set of vertices is the same as that of $\Gamma$ and $x \sim y$ in $\tilde{\Gamma}$ if $d(x, y) \leq 2$ in $\Gamma$. The graph $\tilde{\Gamma}$ is equipped with a weight $\tilde{\mu}$ defined by

$$
\tilde{\mu}_{x y}=p_{2}(x, y) m(x) \text {. }
$$

In other words, the Markov kernel $\tilde{p}(x, y)$ on $\tilde{\Gamma}$ is given by $\tilde{p}(x, y)=p_{2}(x, y)$, and the corresponding measure $\tilde{m}$ coincides with $m$. The heat kernels $h$ and $\tilde{h}$ respectively on $\Gamma$ and $\tilde{\Gamma}$ are related as follows.

LEMmA 3.1. For all $k \in N^{*}$ and $x, y \in \Gamma$, we have

$$
h_{2 k}(x, y)=\tilde{h}_{k}(x, y)
$$

and

$$
h_{2 k+1}(x, y) \leq \max _{z \in B(y, 1)} \tilde{h}_{k}(x, z) .
$$

PROOF. Indeed, we have

$$
h_{2 k}(x, y)=\frac{p_{2 k}(x, y)}{m(y)}=\frac{\tilde{p}_{k}(x, y)}{\tilde{m}(y)}=\tilde{h}_{k}(x, y)
$$


and

$$
h_{2 k+1}(x, y)=\sum_{z \in B(y, 1)} h_{2 k}(x, z) p(y, z) \leq \max _{z \in B(y, 1)} \tilde{h}_{k}(x, z) \sum_{z \in \Gamma} p(y, z)=\max _{z \in B(y, 1)} \tilde{h}_{k}(x, z) .
$$

Most of our results in the next sections use the following lemma.

LEMMA $3.2([9, \S 4.2])$. If $(\Gamma, \mu)$ satisfies Condition $(\beta)$, then $(\tilde{\Gamma}, \tilde{\mu})$ satisfies Condition $(\alpha)$, with $\alpha=1 / \beta$.

Proof. By the Cauchy-Schwarz inequality, for any $x \in \Gamma$,

$$
\begin{aligned}
h_{2}(x, x) & =\sum_{y \in B(x, 1)} h^{2}(x, y) m(y) \\
& \geq \frac{1}{m(B(x, 1))}\left(\sum_{y \in B(x, 1)} h(x, y) m(y)\right)^{2}=\frac{1}{m(B(x, 1))},
\end{aligned}
$$

that is,

$$
\tilde{p}(x, x)=p_{2}(x, x)=h_{2}(x, x) m(x) \geq \frac{m(x)}{m(B(x, 1))},
$$

whence the claim follows.

Using Lemmas 3.1 and 3.2, one can easily formulate some versions of Propositions 2.3 and 2.5 adapted to graphs satisfying Condition $(\beta)$ instead of $(\alpha)$; here the conclusion is that the expressions $J_{k}$ decay separately for even and odd times. We leave details to the reader.

A couple of comments are in order about Condition $(\beta)$. First it is obviously equivalent to the conjunction of the following two properties:

- $\sup _{x \in \Gamma} N_{x}<+\infty$, where $N_{x}$ is the number of neighbors of $x$, that is, the graph $\Gamma$ is locally uniformly finite.

- there exists a positive constant $C$ such that $m(y) / C \leq m(x) \leq C m(y)$ if $x, y$ are neighbors.

Note that, in the case of the simple random walk on $\Gamma$, the second condition follows from the first one.

On the other hand, it is easy to see that either of the following conditions on $(\Gamma, \mu)$ implies Condition $(\beta)$ :

- $\inf _{x \sim y} p(x, y)>0$.

- $(\Gamma, \mu)$ is invariant under a quasi-transitive group action, that is, there exists a group $G$, acting on the graph $\Gamma$ with finitely many orbits, such that $\mu$ is $G$-invariant.

Note finally that neither of Conditions $(\alpha),(\beta)$ implies the other one for the same graph.

4. The Davies-Gaffney estimate of the heat kernel. The aim of this section is to derive the following statement from the discrete integrated maximum principle. Here $(\cdot, \cdot)$ denotes the inner product in $l^{2}(\Gamma, m)$, and $\|\cdot\|_{2}$ is the corresponding norm. 
THEOREM 4.1. Let $(\Gamma, \mu)$ be a weighted graph satisfying either Condition $(\alpha)$ or Condition $(\beta)$. There exist positive constants $C, c$ depending only on $\alpha$ or $\beta$, such that, for any two subsets $A, B \subset \Gamma$, for all $f \in l^{2}(A), g \in l^{2}(B)$, and all $k \in N^{*}$, we have

$$
\left|\left(P^{k} f, g\right)\right| \leq C \exp \left(-c \frac{d^{2}(A, B)}{k}\right)\|f\|_{2}\|g\|_{2},
$$

where $d(A, B):=\inf \{d(x, y) ; x \in A, y \in B\}$.

Proof. We first prove the statement when $(\Gamma, \mu)$ satisfies Condition $(\alpha)$. Setting $u_{k}:=$ $P^{k} f$ we have

$$
\left|\left(P^{k} f, g\right)\right|=\left|\sum_{y \in B} u_{k}(y) g(y) m(y)\right| \leq\left(\sum_{y \in B} u_{k}^{2}(y) m(y)\right)^{1 / 2}\|g\|_{2} .
$$

Then consider the quantity

$$
J_{k}:=\sum_{y \in \Gamma} u_{k}^{2}(y) e^{a d(y, A)-b k} m(y),
$$

with $a \geq 0$ and $b=c(\alpha) a^{2}$ as in Remark 2.4, and record the following three facts:

- Since $u_{0}=f$ is supported in $A$ and $d(\cdot, A) \equiv 0$ on $A$,

$$
J_{0}=\sum_{y \in A} u_{0}^{2}(y) m(y)=\|f\|_{2}^{2} .
$$

- By Proposition 2.3 and Remark 2.4, $J_{k} \leq J_{0}$.

- Since $d(y, A) \geq d(A, B)$ for any $y \in B$,

$$
J_{k} \geq e^{a d(A, B)-b k} \sum_{y \in B} u_{k}^{2}(y) m(y) .
$$

Therefore

$$
\sum_{y \in B} u_{k}^{2}(y) m(y) \leq e^{-a d(A, B)+b k}\|f\|_{2}^{2},
$$

whence, by choosing $a=\lambda d(A, B) / k$, where $\lambda$ is any positive number smaller than $1 / c(\alpha)$,

$$
\sum_{y \in B} u_{k}^{2}(y) m(y) \leq \exp \left(-c \frac{d^{2}(A, B)}{k}\right)\|f\|_{2}^{2} .
$$

Substituting into (4.2), we obtain (4.1).

Assume now that $(\Gamma, \mu)$ satisfies Condition $(\beta)$. Then, by Lemma 3.2, the iterated graph $(\tilde{\Gamma}, \tilde{\mu})$ satisfies Condition $(\alpha)$. By the first part of this proof, we conclude that

$$
\left|\left(\tilde{P}^{k} f, g\right)\right| \leq C \exp \left(-c \frac{\tilde{d}^{2}(A, B)}{k}\right)\|f\|_{2}\|g\|_{2},
$$


where $\tilde{P}$ is the Markov operator on $(\tilde{\Gamma}, \tilde{\mu})$ associated with $\tilde{p}$, and $\tilde{d}$ is the graph distance on $\tilde{\Gamma}$. Since $\tilde{P}^{k}=P^{2 k}$ and $\tilde{d}=\lceil d / 2\rceil \geq d / 2$ (where $\lceil\gamma\rceil$ is the smallest integer larger than or equal to $\gamma$ ), we obtain

$$
\left|\left(P^{2 k} f, g\right)\right| \leq C \exp \left(-\frac{c}{4} \frac{d^{2}(A, B)}{k}\right)\|f\|_{2}\|g\|_{2} .
$$

Finally, noticing that the support of $P f$ is in the 1-neighborhood $A^{\prime}$ of $A$, we obtain from (4.4) applied to $P f$ instead of $f$ :

$$
\begin{aligned}
\left|\left(P^{2 k+1} f, g\right)\right| & \leq C \exp \left(-\frac{c}{4} \frac{d^{2}\left(A^{\prime}, B\right)}{k}\right)\|P f\|_{2}\|g\|_{2} . \\
& \leq C^{\prime} \exp \left(-c^{\prime} \frac{d^{2}(A, B)}{k}\right)\|f\|_{2}\|g\|_{2},
\end{aligned}
$$

where we have also used $\|P f\|_{2} \leq\|f\|_{2}$.

COROLlary 4.2. Let $(\Gamma, \mu)$ be a weighted graph satisfying either Condition $(\alpha)$ or Condition $(\beta)$. There exist positive constants $C, c$ depending only on $\alpha$ or $\beta$, such that, for any two subsets $A, B \subset \Gamma$ with finite measure and for any $k \in N^{*}$, we have

$$
\sum_{x \in A} \sum_{y \in B} h_{k}(x, y) m(x) m(y) \leq C \exp \left(-c \frac{d^{2}(A, B)}{k}\right) \sqrt{m(A) m(B)}
$$

and

$$
\sum_{x \in A} \sum_{y \in B} h_{k}^{2}(x, y) m(x) m(y) \leq C \exp \left(-c \frac{d^{2}(A, B)}{k}\right) \min (\operatorname{card}(A), \operatorname{card}(B)) .
$$

Proof. By taking $f=\mathbf{1}_{A}, g=\mathbf{1}_{B}$ in (4.1) we obtain (4.5). To prove (4.6), we use (4.3) with $f(y)=\delta_{x, y} / m(x)$ with a fixed $x \in \Gamma$. Then $u_{k}(y)=P^{k} f(y)=h_{k}(x, y)$, and (4.3) yields

$$
\sum_{y \in B} h_{k}^{2}(x, y) m(y) \leq \exp \left(-c \frac{d^{2}(x, B)}{k}\right) \frac{1}{m(x)} .
$$

Multiplying by $m(x)$, summing in $x \in A$, and noticing that $d(x, B) \geq d(A, B)$, we obtain

$$
\sum_{x \in A} \sum_{y \in B} h_{k}^{2}(x, y) m(x) m(y) \leq \exp \left(-c \frac{d^{2}(A, B)}{k}\right) \operatorname{card}(A) .
$$

Hence, (4.6) follows by symmetry between $A$ and $B$.

5. From on-diagonal to off-diagonal upper estimates. Let us first recall the notion of regular function introduced in [16].

Definition 5.1. We say that a function $f:(0,+\infty) \rightarrow(0,+\infty)$ is regular if $f$ is monotonically increasing and if there exist $A \geq 1$ and $\gamma>1$ such that for all $0<s<t$ we 
have

$$
\frac{f(\gamma s)}{f(s)} \leq A \frac{f(\gamma t)}{f(t)} .
$$

Here are two (opposite) sufficient conditions for regularity:

(1) Let $f(t)$ satisfy the doubling condition, that is, for some $A \geq 1$ and all $t>0$

$$
f(2 t) \leq A f(t) .
$$

Then $f$ is regular with $\gamma=2$ because

$$
\frac{f(2 s)}{f(s)} \leq A \leq A \frac{f(2 t)}{f(t)} .
$$

(2) Let $f(t)$ have at least polynomial growth in the sense that, for some $\gamma>1$, the function $f(\gamma t) / f(t)$ is increasing in $t$. Then $f$ is regular with $A=1$.

Consider a function $l(\xi):=\log f\left(e^{\xi}\right)$ where $\xi \in(-\infty,+\infty)$. If $f$ is differentiable then regularity is implied by either of the following two conditions:

(1) $l^{\prime}$ is uniformly bounded (for example, this is the case when $f(t)=t^{N}$ or $f(t)=$ $\log ^{N}(1+t)$ where $\left.N>0\right)$;

(2) $l^{\prime}$ is monotone increasing (for example, $f(t)=\exp \left(t^{N}\right)$ ).

On the other hand, regularity fails if $l^{\prime}=\exp (-\xi)$ (that is, unbounded and decreasing), which corresponds to $f(t)=\exp \left(-t^{-1}\right)$. Also, regularity may fail if $l^{\prime}$ is oscillating.

We can now state the main result of this section.

THEOREM 5.2. Let $(\Gamma, \mu)$ be a weighted graph satisfying Condition $(\alpha)$. Let $x, y$ be two fixed vertices in $\Gamma$, and assume that there are two regular functions $f, g$ (satisfying (5.1) with the same constants $\gamma, A$ ) such that, for all $k \in N^{*}$,

$$
\begin{aligned}
& h_{2 k}(x, x) \leq \frac{1}{f(k)}, \\
& h_{2 k}(y, y) \leq \frac{1}{g(k)} .
\end{aligned}
$$

Then, for all $k \in N^{*}$,

$$
h_{k}(x, y) \leq \frac{C_{0}}{\sqrt{f(\eta k) g(\eta k)}} \exp \left(-\frac{d^{2}(x, y)}{D_{0} k}\right),
$$

where $\eta=\eta(\gamma)>0, D_{0}=D_{0}(\alpha, \gamma)>0$ and $C_{0}=C_{0}(A, \alpha, \gamma)$.

If $(\Gamma, \mu)$ satisfies Condition $(\beta)$ instead of $(\alpha)$ then the conclusion (5.3) still holds but only for even $k$, and $C_{0}, D_{0}$ depend on $\beta$ instead of $\alpha$.

To prove the above result, we shall follow closely the strategy of [16]. For $D>0, x \in \Gamma$, consider the following quantity:

$$
E_{D}(k, x):=\sum_{z \in \Gamma} h_{k}^{2}(x, z) \exp \left(\frac{d_{1}^{2}(x, z)}{D k}\right) m(z),
$$


where

$$
d_{1}(x, z):=\max \{d(x, z), 1\} .
$$

Note that $E_{D}(k, x) \rightarrow h_{2 k}(x, x)$ as $D \rightarrow \infty$.

Proposition 5.3. Let $(\Gamma, \mu)$ be a weighted graph. For all $x, y \in \Gamma, k \in N^{*}$, and all $D>0$,

$$
h_{2 k}(x, y) \leq \sqrt{E_{D}(k, x) E_{D}(k, y)} \exp \left(-\frac{d^{2}(x, y)}{4 D k}\right) .
$$

Proof. By the Cauchy-Schwarz inequality, since $d_{1}^{2}(x, y) \leq 2\left(d_{1}^{2}(x, z)+d_{1}^{2}(y, z)\right)$, we have

$$
\begin{aligned}
h_{2 k}(x, y) & \leq \sum_{z \in \Gamma} h_{k}(x, z) h_{k}(y, z) e^{d_{1}^{2}(x, z) / 2 D k} e^{d_{1}^{2}(y, z) / 2 D k} e^{-d_{1}^{2}(x, y) / 4 D k} m(z) \\
& \leq \sqrt{E_{D}(k, x) E_{D}(k, y)} e^{-d_{1}^{2}(x, y) / 4 D k} .
\end{aligned}
$$

Since $d_{1}(x, y) \geq d(x, y)$, the claim is proved.

Observe that, as $D \rightarrow+\infty$, equation (5.5) becomes the well-known estimate $h_{2 k}(x, y) \leq$ $\sqrt{h_{2 k}(x, x) h_{2 k}(y, y)}$.

Proposition 5.3 enables one to obtain an upper bound for $h_{2 k}(x, y)$ from an upper bound for $E_{D}(k, y)$. More precisely, Theorem 5.2 is an obvious consequence of (5.5) and the following statement.

Proposition 5.4. Let $(\Gamma, \mu)$ be a weighted graph satisfying Condition $(\alpha)$. Let $x$ be a fixed vertex in $\Gamma$, and assume that there exists a regular function $f$ such that, for all $k \in N^{*}$,

$$
h_{2 k}(x, x) \leq \frac{1}{f(k)} .
$$

Then, for all $k \in N^{*}$,

$$
E_{D_{0}}(k, x) \leq \frac{C_{0}}{f(\eta k)}
$$

where $\eta=\eta(\gamma)>0, D_{0}=D_{0}(\alpha, \gamma)>0$, and $C_{0}=C_{0}(A, \alpha, \gamma)$ (here $\gamma, A$ are the constants from (5.1)).

The same statement is true if $(\Gamma, \mu)$ satisfies Condition $(\beta)$ instead of $(\alpha)$, with the constants $D_{0}$ and $C_{0}$ depending on $\beta$ instead of $\alpha$.

Proof of TheOrem 5.2. Using (5.5) with $D=D_{0}$ and applying Proposition 5.4, we obtain

$$
\begin{aligned}
h_{2 k}(x, y) & \leq \sqrt{E_{D}(k, x) E_{D}(k, y)} \exp \left(-\frac{d^{2}(x, y)}{4 D k}\right) \\
& \leq \frac{C_{0}}{\sqrt{f(\eta k) g(\eta k)}} \exp \left(-\frac{d^{2}(x, y)}{4 D k}\right),
\end{aligned}
$$

which yields (5.3) for even times. 
If $(\Gamma, \mu)$ satisfies Condition $(\alpha)$, then we write

$$
h_{2 k}(x, y)=\sum_{z \in B(y, 1)} h_{2 k-1}(x, z) p(y, z) \geq h_{2 k-1}(x, y) p(y, y) \geq \alpha h_{2 k-1}(x, y),
$$

whence

$$
h_{2 k-1}(x, y) \leq \alpha^{-1} h_{2 k}(x, y) .
$$

Substituting the estimate (5.8), we obtain (5.3) for odd times.

Proof of Proposition 5.4. Let us first assume that $(\Gamma, \mu)$ satisfies Condition $(\alpha)$. Fix $x \in \Gamma$ and, for any $R>0$ and $k \in N$, define

$$
I(R, k)=I(k, x, R):=\sum_{z \notin B(x, R)} h_{k}^{2}(x, z) m(z) .
$$

We will estimate $I(R, k)$ by iteration, and the iterative step is contained in the following estimate: for all $n, k \in N$ such that $n>k$ and all real numbers $R \geq r>0$,

$$
I(R, n) \leq\left(h_{2 k}(x, x) \exp \left(-\frac{(R-r)^{2}}{2 D_{\alpha}(n-k)}\right)+I(r, k)\right) \exp \left(1 / D_{\alpha}\right),
$$

where $D_{\alpha}$ is the constant from Proposition 2.5. Denote by $\lfloor R\rfloor$ the integer part of $R$. Define

$$
\rho(z):= \begin{cases}d\left(z, B(x, R)^{c}\right)+1=\lfloor R\rfloor-d(x, z)+2, & \text { if } z \in B(x, R), \\ 1, & \text { otherwise. }\end{cases}
$$

Note that $\rho$ satisfies the assumptions of Proposition 2.5. Let

$$
F_{k}(x):=\exp \left(-\frac{\rho^{2}(x)}{D_{\alpha}(n+1-k)}\right), k=0, \ldots, n .
$$

Since $F_{k}(z) \geq \exp \left(-1 / D_{\alpha}\right)$ for $z \notin B(x, R)$, we can write

$$
I(R, n)=\sum_{z \notin B(x, R)} h_{n}^{2}(x, z) m(z) \leq \exp \left(1 / D_{\alpha}\right) \sum_{z \in \Gamma} h_{n}^{2}(x, z) F_{n}(z) m(z) .
$$

Then, we know from Proposition 2.5 that

$$
\sum_{z \in \Gamma} h_{n}^{2}(x, z) F_{n}(z) m(z) \leq \sum_{z \in \Gamma} h_{k}^{2}(x, z) F_{k}(z) m(z) .
$$

If $z \in B(x, r)$, then $\rho(z) \geq\lfloor R\rfloor-r+2 \geq R-r$, whence

$$
\begin{aligned}
\sum_{z \in \Gamma} h_{k}^{2}(x, z) F_{k}(z) m(z) & =\sum_{z \in B(x, r)} h_{k}^{2}(x, z) F_{k}(z) m(z)+\sum_{z \notin B(x, r)} h_{k}^{2}(x, z) F_{k}(z) m(z) \\
& \leq\left(\sum_{z \in B(x, r)} h_{k}^{2}(x, z) m(z)\right) \exp \left(-\frac{(R-r)^{2}}{D_{\alpha}(n+1-k)}\right)+I(r, k) \\
& \leq h_{2 k}(x, x) \exp \left(-\frac{(R-r)^{2}}{2 D_{\alpha}(n-k)}\right)+I(r, k),
\end{aligned}
$$


that is, (5.9). Using the hypothesis (5.6), we obtain from (5.9)

$$
I(R, n) \leq \frac{\exp \left(1 / D_{\alpha}\right)}{f(k)} \exp \left(-\frac{(R-r)^{2}}{2 D_{\alpha}(n-k)}\right)+I(r, k) \exp \left(1 / D_{\alpha}\right) .
$$

Now let us prove that there exist positive numbers $R_{0}=R_{0}(\gamma)$ and $\theta=\theta(\gamma)$ such that, for all $R \geq R_{0}$ and $k \in N^{*}$,

$$
I(R, k) \leq \frac{C_{0}}{f(k / \gamma)} \exp \left(-\theta \frac{R^{2}}{k}\right) .
$$

The result is trivial if $R \geq k$ (since then $I(R, k)=0$ ), hence we can suppose $k>R$. Given any finite decreasing sequence $\left\{R_{j}\right\}_{j=1}^{j_{0}}$ of real numbers and any finite strictly decreasing sequence $\left\{k_{j}\right\}_{j=1}^{j_{0}}$ of natural numbers such that $R_{1}=R, k_{1}=k$ and $I\left(R_{j_{0}}, k_{j_{0}}\right)=0$, we can iterate (5.10) and obtain

$$
I(R, k) \leq \sum_{j=1}^{j_{0}-1} \frac{\exp \left(j / D_{\alpha}\right)}{f\left(k_{j+1}\right)} \exp \left(-\frac{\left(R_{j}-R_{j+1}\right)^{2}}{2 D_{\alpha}\left(k_{j}-k_{j+1}\right)}\right) .
$$

Let us now build such sequences $\left\{R_{j}\right\}_{j=1}^{j_{0}}$ and $\left\{k_{j}\right\}_{j=1}^{j_{0}}$. Recall that $\gamma>1$. Take $R>R_{0}$, where

$$
R_{0}=R_{0}(\gamma):=2 \gamma /(\gamma-1)+2,
$$

and

$$
R_{j}:=R / 2+R /(j+1), \quad t_{j}:=k / \gamma^{j-1}, \quad k_{j}:=\left\lceil t_{j}\right\rceil .
$$

Let $j_{0}:=\min \left\{j ; R_{j} \geq k_{j}\right\}$ (note that $j_{0}>1$ since $k>R$ ). By construction, one has $I\left(R_{j_{0}}, k_{j_{0}}\right)=0$. Also, for all $j<j_{0}$ we have $k_{j}>R_{j}>R / 2$, whence

$$
t_{j}-t_{j+1}=t_{j}\left(1-\frac{1}{\gamma}\right) \geq\left(k_{j}-1\right)\left(1-\frac{1}{\gamma}\right) \geq(R / 2-1)\left(1-\frac{1}{\gamma}\right)>1,
$$

which means that $k_{j}>k_{j+1}$. Moreover,

$$
k_{j}-k_{j+1} \leq k / \gamma^{j-1}-k / \gamma^{j}+1=k(\gamma-1) / \gamma^{j}+1 \leq 2 k(\gamma-1) / \gamma^{j},
$$

where in the last inequality we used the fact that $k>\gamma^{j} /(\gamma-1)$ which follows from $k_{j}>R / 2$ and the choice of $R$. Using the estimate for $k_{j}-k_{j+1}$ and the identity

we obtain

$$
\left(R_{j}-R_{j+1}\right)^{2}=\frac{R^{2}}{(j+1)^{2}(j+2)^{2}},
$$

$$
\frac{\left(R_{j}-R_{j+1}\right)^{2}}{2 D_{\alpha}\left(k_{j}-k_{j+1}\right)} \geq \theta \frac{R^{2}}{k}(j+1)
$$

where

$$
\theta=\theta(\alpha, \gamma):=\frac{1}{4 D_{\alpha}(\gamma-1)} \min _{j \geq 1} \frac{\gamma^{j}}{(j+1)^{3}(j+2)^{2}}>0
$$


Therefore (5.12) yields

$$
I(R, k) \leq \sum_{j=1}^{j_{0}-1} \frac{1}{f\left(t_{j+1}\right)} \exp \left(\frac{j}{D_{\alpha}}-\theta \frac{R^{2}}{k}(j+1)\right) .
$$

By the regularity of $f$, we have

$$
\frac{f\left(t_{j}\right)}{f\left(t_{j+1}\right)}=\frac{f\left(\gamma t_{j+1}\right)}{f\left(t_{j+1}\right)} \leq A \frac{f\left(t_{1}\right)}{f\left(t_{2}\right)}=A \frac{f(k)}{f(k / \gamma)},
$$

whence

$$
\frac{f\left(t_{1}\right)}{f\left(t_{j+1}\right)}=\frac{f\left(t_{1}\right)}{f\left(t_{2}\right)} \frac{f\left(t_{2}\right)}{f\left(t_{3}\right)} \cdots \frac{f\left(t_{j}\right)}{f\left(t_{j+1}\right)} \leq\left(A \frac{f(k)}{f(k / \gamma)}\right)^{j} .
$$

Thus, setting $L:=\log (A f(k) / f(k / \gamma))$,

$$
\frac{1}{f\left(t_{j+1}\right)} \leq \frac{1}{f\left(t_{1}\right)} \exp (j L)=\frac{1}{f(k)} \exp (j L),
$$

and

$$
I(R, k) \leq \frac{1}{f(k)} \exp \left(-\theta \frac{R^{2}}{k}\right) \sum_{j=1}^{j_{0}-1} \exp \left(-j\left(\theta \frac{R^{2}}{k}-L-\frac{1}{D_{\alpha}}\right)\right) .
$$

We consider two cases:

CASE 1: Let

$$
\theta \frac{R^{2}}{k}-L-\frac{1}{D_{\alpha}} \geq \log 2
$$

In this case we have

$$
I(R, k) \leq \frac{1}{f(k)} \exp \left(-\theta \frac{R^{2}}{k}\right) \sum_{j=1}^{j_{0}-1} 2^{-j} \leq \frac{1}{f(k)} \exp \left(-\theta \frac{R^{2}}{k}\right) .
$$

CASE 2: Let

$$
\theta \frac{R^{2}}{k}-L-\frac{1}{D_{\alpha}}<\log 2 .
$$

In this case we estimate $I(R, k)$ differently:

$$
\begin{aligned}
I(R, k) & \leq \sum_{z \in \Gamma} h_{k}^{2}(x, z) m(z)=h_{2 k}(x, x) \leq \frac{1}{f(k)} \\
& \leq \frac{2}{f(k)} \exp \left(L+\frac{1}{D_{\alpha}}-\theta \frac{R^{2}}{k}\right)=\frac{2 A \exp \left(1 / D_{\alpha}\right)}{f(k / \gamma)} \exp \left(-\theta \frac{R^{2}}{k}\right) .
\end{aligned}
$$

In both cases we have

$$
I(R, k) \leq \frac{2 A \exp \left(1 / D_{\alpha}\right)}{f(k / \gamma)} \exp \left(-\theta \frac{R^{2}}{k}\right)
$$

for all $R \geq R_{0}(\gamma)$, which is (5.11). 
Finally, let us prove (5.7). Define, for $j \in N$,

$$
\mathcal{A}_{j}^{R}:= \begin{cases}\left\{z \in \Gamma ; d_{1}(x, z) \leq R\right\}, & j=0 \\ \left\{z \in \Gamma ; 2^{j-1} R<d_{1}(x, z) \leq 2^{j} R\right\}, & j \geq 1\end{cases}
$$

and

$$
E_{D}(k, x)=\sum_{j=0}^{\infty} \sum_{z \in \mathcal{A}_{j}^{R}} h_{k}^{2}(x, z) \exp \left(\frac{d_{1}^{2}(x, z)}{D k}\right) m(z) .
$$

For any $D>0$, the first term of this sum admits the estimate

$$
\sum_{z \in \mathcal{A}_{0}^{R}} h_{k}^{2}(x, z) \exp \left(\frac{d_{1}^{2}(x, z)}{D k}\right) m(z) \leq h_{2 k}(x, x) \exp \left(\frac{R^{2}}{D k}\right) \leq \frac{1}{f(k)} \exp \left(\frac{R^{2}}{D k}\right) .
$$

Now for the remaining terms we have, assuming $R \geq 1$,

$$
\sum_{z \in \mathcal{A}_{j}^{R}} h_{k}^{2}(x, z) \exp \left(\frac{d_{1}^{2}(x, z)}{D k}\right) m(z) \leq \exp \left(\frac{4^{j} R^{2}}{D k}\right) I\left(2^{j-1} R, k\right) .
$$

Assuming $R \geq R_{0}(\gamma)$, we obtain by (5.11)

$$
I\left(2^{j-1} R, k\right) \leq \frac{C_{0}}{f(k / \gamma)} \exp \left(-\theta \frac{4^{j-1} R^{2}}{k}\right),
$$

whence

$$
\begin{aligned}
\sum_{z \in \mathcal{A}_{j}^{R}} h_{k}^{2}(x, z) \exp \left(\frac{d_{1}^{2}(x, z)}{D k}\right) m(z) & \leq \exp \left(\frac{4^{j} R^{2}}{D k}\right) \frac{C_{0}}{f(k / \gamma)} \exp \left(-\theta \frac{4^{j-1} R^{2}}{k}\right) \\
& \leq \frac{C_{0}}{f(k / \gamma)} \exp \left(-\frac{4^{j-1} R^{2}}{D k}\right),
\end{aligned}
$$

provided $D \geq 5 / \theta$.

Take

$$
D_{0}=D_{0}(\alpha, \gamma):=\max \left(\frac{5}{\theta}, \frac{R_{0}^{2}}{\log 2}\right) .
$$

Then by (5.13) and (5.14) we obtain, for any $R \geq R_{0}$,

$$
E_{D_{0}}(k, x) \leq \frac{1}{f(k)} \exp \left(\frac{R^{2}}{D_{0} k}\right)+\frac{C_{0}}{f(k / \gamma)} \sum_{j=1}^{\infty} \exp \left(-\frac{4^{j-1} R^{2}}{D_{0} k}\right) .
$$

Given $k \in N^{*}$ choose $R$ so that $R^{2} /\left(D_{0} k\right)=\log 2$. Since by (5.15) $R \geq R_{0}$, we conclude

$$
E_{D_{0}}(k, x) \leq \frac{2}{f(k)}+\frac{C_{0}}{f(k / \gamma)} \sum_{j=1}^{\infty} 2^{-4^{j-1}} \leq \frac{2+C_{0}}{f(k / \gamma)}
$$

which was to be proved. 
Now let us consider the case where $(\Gamma, \mu)$ satisfies Condition $(\beta)$. The hypothesis (5.6) means that for the heat kernel $\tilde{h}_{k}$ on the iterated graph $(\tilde{\Gamma}, \tilde{\mu})$ we have

$$
\tilde{h}_{k}(x, x) \leq \frac{1}{f(k)} .
$$

Since $(\tilde{\Gamma}, \tilde{\mu})$ satisfies Condition $(\alpha)$, the above proof yields, for any $D \geq D_{0}$,

$$
\tilde{E}_{D}(k, x) \leq \frac{C_{0}}{f(k / \gamma)}
$$

where

$$
\tilde{E}_{D}(k, x)=\sum_{z \in \Gamma} \tilde{h}_{k}^{2}(x, z) \exp \left(\frac{\tilde{d}_{1}^{2}(x, z)}{D k}\right) m(z) .
$$

Clearly,

$$
\tilde{E}_{D}(k, x) \geq \sum_{z \in \Gamma} h_{2 k}^{2}(x, z) \exp \left(\frac{d_{1}^{2}(x, z)}{4 D k}\right) m(z)=E_{4 D}(2 k, x),
$$

which together with (5.18) yields

$$
E_{4 D}(2 k, x) \leq \frac{C_{0}}{f(k / \gamma)}
$$

To treat odd $k$, we start with the inequality

$$
h_{2 k+1}^{2}(x, z) \leq \max _{y \in B(z, 1)} h_{2 k}^{2}(x, y) \leq \sum_{y \in B(z, 1)} h_{2 k}^{2}(x, y) .
$$

Since Condition $(\beta)$ implies that $m(z) \leq \beta m(y)$ for all $y \sim z$ and $N_{y} \leq \beta^{2}$ for all $y \in \Gamma$, we can write

$$
\begin{aligned}
E_{8 D}(2 k+1, x) & \leq \sum_{z \in \Gamma} \sum_{y \in B(z, 1)} h_{2 k}^{2}(x, y) \exp \left(\frac{d_{1}^{2}(x, z)}{8 D k}\right) m(z) \\
& \leq \beta \sum_{z \in \Gamma} \sum_{y \in B(z, 1)} h_{2 k}^{2}(x, y) \exp \left(\frac{d_{1}^{2}(x, y)+1}{4 D k}\right) m(y) \\
& \leq \beta e^{1 / 4 D} \sum_{y \in \Gamma} \sum_{z \in B(y, 1)} h_{2 k}^{2}(x, y) \exp \left(\frac{d_{1}^{2}(x, y)}{4 D k}\right) m(y) \\
& =\beta e^{1 / 4 D} N_{y} E_{4 D}(2 k, x) \leq \beta^{3} e^{1 / 4 D} E_{4 D}(2 k, x),
\end{aligned}
$$

whence by (5.19)

$$
E_{8 D}(2 k+1, x) \leq \frac{C(\beta, D) C_{0}}{f(k / \gamma)} .
$$

Combining (5.19) and (5.20) and changing appropriately the constants $C_{0}, D_{0}$, we obtain (5.7) again. 
6. From on-diagonal upper to on-diagonal lower estimates. The aim of this section is to prove the following theorem.

THEOREM 6.1. Let $(\Gamma, \mu)$ be a weighted graph which satisfies Condition $(\alpha)$ and $x \in \Gamma$ a fixed vertex. Let $v$ be a non-decreasing function on $(0,+\infty)$ such that

$$
m(B(x, r)) \leq v(r), \quad r>0
$$

and, for some $A>0$,

$$
v(2 r) \leq A v(r), \quad r>0 .
$$

If there exists a constant $C>0$ such that

$$
h_{k}(x, x) \leq \frac{C}{v(\sqrt{k})}, \quad k \in N^{*},
$$

then

$$
h_{k}(x, x) \geq \frac{c}{v(\sqrt{k})}, \quad k \in N^{*},
$$

for some $c=c(A, C, \alpha)>0$.

If $(\Gamma, \mu)$ satisfies Condition $(\beta)$ instead of $(\alpha)$ then the conclusion (6.4) still holds but only for even $k$, and $c$ depends on $\beta$ instead of $\alpha$.

We note that under Condition $(\beta)$ alone we cannot hope to extend the estimate (6.4) to odd values of time since it may happen that $h_{2 k+1}(x, x)=0$.

We start with a lemma, which is well-known in the context of continuous-time heat kernels (see for example [23]).

LeMmA 6.2. Let $(\Gamma, \mu)$ be a weighted graph and $x \in \Gamma$ be a fixed vertex. Let $\Omega$ be a non-empty subset of $\Gamma$. If, for some $\varepsilon>0$ and $k \in N^{*}$,

$$
\sum_{y \in \Gamma \backslash \Omega} h_{k}(x, y) m(y) \leq \varepsilon,
$$

then

$$
h_{2 k}(x, x) \geq \frac{(1-\varepsilon)^{2}}{m(\Omega)} .
$$

Proof. Indeed, using (1.8) and the Cauchy-Schwarz inequality, one has

$$
h_{2 k}(x, x) \geq \sum_{y \in \Omega} h_{k}^{2}(x, y) m(y) \geq \frac{1}{m(\Omega)}\left(\sum_{y \in \Omega} h_{k}(x, y) m(y)\right)^{2}=\frac{(1-\varepsilon)^{2}}{m(\Omega)} .
$$

Since $h_{k}(x, y)=0$ if $y \notin B(x, k)$, this lemma implies immediately the following universal on-diagonal lower bound for the heat kernel

$$
h_{2 k}(x, x) \geq \frac{1}{m(B(x, k))} .
$$


Proof. Let us fix $k \in N^{*}$ and recall the definition

$$
E_{D_{0}}(k, x):=\sum_{y \in \Gamma} h_{k}^{2}(x, y) \exp \left(\frac{d_{1}^{2}(x, y)}{D_{0} k}\right) m(y),
$$

where $D_{0}$ is as in Proposition 5.4. Given any $R \geq 0$ we have, using the Cauchy-Schwarz inequality and $d \leq d_{1}$,

$$
\begin{aligned}
\left(\sum_{y \in \Gamma \backslash B(x, R)} h_{k}(x, y) m(y)\right)^{2} & \leq E_{D_{0}}(k, x) \sum_{y \in \Gamma \backslash B(x, R)} \exp \left(-\frac{d^{2}(x, y)}{D_{0} k}\right) m(y) \\
& =E_{D_{0}}(k, x) S(k, x, R),
\end{aligned}
$$

where

$$
S(k, x, R):=\sum_{y \in \Gamma \backslash B(x, R)} \exp \left(-\frac{d^{2}(x, y)}{D_{0} k}\right) m(y) .
$$

Our aim is to find some $R>0$ such that the expression in (6.5) is smaller than, say, $1 / 2$ and then we shall apply Lemma 6.2.

The function $t \mapsto v(\sqrt{t})$ is clearly regular with constants $\gamma=4$ and $A$. Assuming that $(\Gamma, \mu)$ satisfies $(\alpha)$ or $(\beta)$, Proposition 5.4 yields

$$
E_{D_{0}}(k, x) \leq \frac{C_{0}}{v(\sqrt{k})}
$$

Let us estimate $S(k, x, R)$ as follows, using the notation $R_{j}:=2^{j} R$ :

$$
\begin{aligned}
S(k, x, R) & \leq \sum_{j=0}^{+\infty} \exp \left(-\frac{R_{j}^{2}}{D_{0} k}\right) m\left(B\left(x, R_{j+1}\right) \backslash B\left(x, R_{j}\right)\right) \\
& \leq \sum_{j=0}^{+\infty} \exp \left(-\frac{R_{j}^{2}}{D_{0} k}\right) m\left(B\left(x, R_{j+1}\right)\right) \\
& \leq \sum_{j=0}^{+\infty} \exp \left(-\frac{R_{j}^{2}}{D_{0} k}+\log \left(v\left(R_{j+1}\right)\right)\right),
\end{aligned}
$$

where in the last inequality we used (6.1). From (6.2) we have that $v\left(R_{j+1}\right) \leq A^{j+1} v(R)$, and since $4^{j} \geq j+1$ for any $j \in N$, we have

$$
\begin{aligned}
S(k, x, R) & \leq \sum_{j=0}^{+\infty} \exp \left(-4^{j} \frac{R^{2}}{D_{0} k}+\log (v(R))+(j+1) \log A\right) \\
& \leq v(R) \sum_{j=1}^{+\infty} \exp \left(-j\left(\frac{R^{2}}{D_{0} k}-\log A\right)\right) .
\end{aligned}
$$


Finally we take $R=a \sqrt{D_{0} k}$ with $a^{2} \geq 2 \log A$, obtaining

$$
S(k, x, R) \leq v(R) \sum_{j=1}^{+\infty} \exp \left(-j \frac{a^{2}}{2}\right)=\frac{v(R)}{\exp \left(a^{2} / 2\right)-1} .
$$

Hence (6.5), (6.7) and (6.8) yield

$$
\left(\sum_{y \in \Gamma \backslash B(x, R)} h_{k}(x, y) m(y)\right)^{2} \leq \frac{C_{0}}{v(\sqrt{k})} \frac{v(R)}{\exp \left(a^{2} / 2\right)-1} \leq \frac{C_{0} A^{\log _{2}\left(a \sqrt{D_{0}}\right)+1}}{\exp \left(a^{2} / 2\right)-1},
$$

where we used repeatedly (6.2) to obtain

$$
\frac{v(R)}{v(\sqrt{k})} \leq A^{\log _{2}\left(a \sqrt{D_{0}}\right)+1} .
$$

There exists large enough $a_{0}$ such that

$$
\frac{C_{0} A^{\log _{2}\left(a_{0} \sqrt{D_{0}}\right)+1}}{\exp \left(a_{0}^{2} / 2\right)-1} \leq \frac{1}{4} .
$$

Then Lemma 6.2 implies

$$
h_{2 k}(x, x) \geq \frac{1 / 4}{m\left(B\left(x, a_{0} \sqrt{D_{0} k}\right)\right)} \geq \frac{1 / 4}{v\left(a_{0} \sqrt{D_{0} k}\right)} \geq \frac{c}{v(\sqrt{2 k})},
$$

where

$$
c:=\frac{1 / 4}{A^{\log _{2}\left(a_{0} \sqrt{D_{0} / 2}\right)+1}} .
$$

This finishes the proof of (6.4) for even $k$. If $(\Gamma, \mu)$ satisfies $(\alpha)$ then by $(1.8)$ and $(\alpha)$ one has

$$
h_{2 k+1}(x, x)=\sum_{z \in B(x, 1)} h_{2 k}(x, z) p(x, z) \geq h_{2 k}(x, x) p(x, x) \geq \frac{\alpha c}{v(\sqrt{2 k})} \geq \frac{\alpha c}{v(\sqrt{2 k+1})},
$$

which was to be proved.

7. Time-dependent random walks. Our results apply, with minor modifications, to non-autonomous heat equations, in other words to time-dependent random walks. Here the weight $\mu$ depends on time, that is, we consider a sequence $\left(\mu^{(k)}\right)_{k \in N^{*}}$ of weights such that the total weight at each vertex is constant:

$$
m(x)=\sum_{y \in \Gamma} \mu_{x y}^{(k)}, \quad k \in N^{*}, \quad x \in \Gamma .
$$

In other words, we consider a time-dependent Markov chain, reversible with respect to a fixed measure $m$, with transition probability

$$
p^{(k)}(x, y):=\frac{\mu_{x y}^{(k)}}{m(x)}
$$

at time $k$. We shall call $(\Gamma, \mu)=\left(\Gamma,\left(\mu^{(k)}\right)_{k \in N^{*}}\right)$ a time-dependent weighted graph. 
The associated time-dependent discrete heat equation is given by

$$
\partial_{k} u=\Delta^{(k)} u,
$$

where

$$
\Delta^{(k)} u(x):=\sum_{y \in \Gamma} p^{(k)}(x, y)(u(y)-u(x)) .
$$

The unique solution of this equation, given the initial state $u_{0}=\phi$, is given by

$$
u_{k}(x)=\sum_{y \in \Gamma} p_{k}(x, y) \phi(y)
$$

where $p_{k}(x, y)$ is defined recursively by

$$
\begin{aligned}
p_{0}(x, y) & :=\delta_{x, y} \\
p_{k}(x, y) & :=\sum_{z \in \Gamma} p_{k-1}(x, z) p^{(k)}(z, y) .
\end{aligned}
$$

One defines accordingly the heat kernel $h_{k}(x, y):=p_{k}(x, y) / m(y)$.

Note that

$$
\left|\nabla^{(k)} f\right|(x):=\left(\sum_{y \in \Gamma}(f(y)-f(x))^{2} p^{(k)}(x, y)\right)^{1 / 2}
$$

is also time-dependent.

The proof of Theorem 2.2 extends verbatim to this setting, by adding superscripts ${ }^{(k)}$ in the proper places. As a consequence, if $(\Gamma, \mu)=\left(\Gamma,\left(\mu^{(k)}\right)_{k \in N^{*}}\right)$ satisfies

$$
p^{(k)}(x, x) \geq \alpha_{k}>0, \quad k \in N^{*},
$$

then the condition

$$
\partial_{k} f(x)+\frac{\left|\nabla^{(k+1)} f_{k+1}\right|^{2}}{4 \alpha_{k+1} f_{k+1}}(x) \leq 0, \quad x \in \Gamma, \quad k \in[0, n),
$$

implies that $J_{k+1} \leq J_{k}$ for any $k \in[0, n)$, where $J_{k}:=\sum_{x \in \Gamma} u_{k}^{2}(x) f_{k}(x) m(x)$ and $u$ is a solution of (7.1) in $[0, n)$.

If we generalize Condition $(\alpha)$ by saying that a time-dependent $(\Gamma, \mu)$ satisfies it if

$$
\inf _{k \in N^{*}} \inf _{x \in \Gamma} p^{(k)}(x, x)=\alpha>0,
$$

then the statements of Propositions 2.3 and 2.5 also extend verbatim.

One can also extend the construction in Section 3. Given $(\Gamma, \mu)=\left(\Gamma,\left(\mu^{(k)}\right)_{k \in N^{*}}\right)$, define $(\tilde{\Gamma}, \tilde{\mu})=\left(\Gamma,\left(\tilde{\mu}^{(k)}\right)_{k \in N^{*}}\right)$, in the following way: $\tilde{\Gamma}$ is as in Section 3 and $\tilde{\mu}_{x, y}^{(k)}=$ $\tilde{p}^{(k)}(x, y) m(x)$, where $\tilde{p}^{(k)}$ is the Markov kernel defined for $k \in \boldsymbol{N}^{*}$ by

$$
\tilde{p}^{(k)}=\sum_{z \in \Gamma} p^{(2 k)}(x, z) p^{(2 k-1)}(z, y) .
$$

Of course the measure associated to the weights $\tilde{\mu}_{x, y}^{(k)}$ is still independent of $k$ and equal to $m$. 
Then one defines $\tilde{p}_{k}$ and $\tilde{h}_{k}$ from $\tilde{p}^{(k)}$ in a way similar to the way $p_{k}$ and $h_{k}$ were defined from $p^{(k)}$. A trivial adaptation of Lemma 3.1 shows that $(\tilde{\Gamma}, \tilde{\mu})$ satisfies Condition $(\beta)$ if $(\Gamma, \mu)$ satisfies $(\alpha)$ in the above sense.

Theorems 5.2 and 6.1 generalize easily to the time-dependent setting.

As an application, one can for instance prove a result that was conjectured in [18, p. 680]. We shall say that the time-dependent weighted graph $(\Gamma, \mu)$ satisfies uniformly a Sobolev inequality of dimension $N>2$ if there exists $C>0$ such that

$$
\left(\sum_{x \in \Gamma} f(x)^{q} m(x)\right)^{2 / q} \leq C \sum_{x, y \in \Gamma}(f(x)-f(y))^{2} \mu_{x y}^{(k)},
$$

for every function $f$ on $\Gamma$ with finite support and all $k \in N^{*}$, where $q=2 N /(N-2)$.

THEOREM 7.1. Let $(\Gamma, \mu)$ be a time-dependent weighted graph satisfying Condition $(\alpha)$ or Condition $(\beta)$. Assume that $(\Gamma, \mu)$ satisfies uniformly a Sobolev inequality of dimension $N>2$. Then

$$
h_{2 k}(x, y) \leq C k^{-N / 2} \exp \left(-c \frac{d^{2}(x, y)}{k}\right), \quad \text { for all } x, y \in \Gamma, k \in N^{*}
$$

and

$$
h_{2 k}(x, x) \geq c k^{-N / 2}, \text { for all } x \in \Gamma, k \in N^{*} .
$$

PROOF. According to [25, chap. VII],

$$
h_{2 k}(x, x) \leq C k^{-N / 2}, \quad \text { for all } x \in \Gamma .
$$

Then one applies Theorem 5.2 to obtain the first assertion. The second one follows from 6.1 or directly from the first one as in [9, Theorem 6.1].

One can generalize the above statement to $N>0$ by considering Nash inequalities instead of Sobolev inequalities.

An interesting direction for future work would be to devise time-dependent versions of [9] and [2], in order to obtain non-uniform upper estimates

$$
h_{2 k}(x, y) \leq \frac{C}{V(x, \sqrt{k})} \exp \left(-c \frac{d^{2}(x, y)}{k}\right),
$$

as well as the matching off-diagonal lower bounds.

8. Random walks on percolation clusters. A percolation cluster is an infinite connected graph $(\Gamma, \mu)$, which is a subgraph of $\boldsymbol{Z}^{N}$ (with the standard weight) obtained by a certain random procedure. We do not go much into the details of the construction. Our aim is just to point out how certain known results on random walk on such graphs can be selfimproved using Theorem 5.2. It is known that, under certain hypotheses, the heat kernel on a percolation cluster satisfies the following estimate: for any $x \in \Gamma$ there exist positive constants $C_{x}$ and $K_{x}$ such that

$$
h_{2 k}(x, x) \leq C_{x} k^{-N / 2} \text { for all } k \geq K_{x}
$$


(see [20] and also [21] for a continuous time analogue, and [4] for full Gaussian upper and lower bounds for a continuous time random walk on percolation clusters). First note that $(\Gamma, \mu)$ being a connected subgraph of $\boldsymbol{Z}^{N}$ satisfies Condition $(\beta)$ with $\beta=\beta(N)$. Let us also recall the well-known result (see for example [6]) that

$$
h_{2 k}(x, x) \leq B k^{-1 / 2} \text { for all } x \in \Gamma, k \in N^{*},
$$

with a universal constant $B>0$. Fix $x \in \Gamma$ and set

$$
f_{x}(t)= \begin{cases}C_{x}^{-1} t^{N / 2}, & t \geq K_{x}, \\ B^{-1} t^{1 / 2}, & t<K_{x},\end{cases}
$$

so that we obtain from (8.1) and (8.2)

$$
h_{2 k}(x, x) \leq \frac{1}{f_{x}(k)} \quad \text { for all } x \in \Gamma, k \in N^{*} .
$$

Clearly, there exist positive constants $b_{x}$ and $c_{x}$ such that

$$
c_{x} \leq C_{x}^{-1}, \quad b_{x} \leq B^{-1}, \quad c_{x} K_{x}^{N / 2}=b_{x} K_{x}^{1 / 2} .
$$

Set

$$
\tilde{f}_{x}(t)= \begin{cases}c_{x} t^{N / 2}, & t \geq K_{x} \\ b_{x} t^{1 / 2}, & t<K_{x}\end{cases}
$$

so that $\tilde{f}_{x}$ is a regular function with the regularity constants $\gamma=2$ and $A=A(N)$. Since $\tilde{f}_{x} \leq f_{x},(8.3)$ implies

$$
h_{2 k}(x, x) \leq \frac{1}{\tilde{f}_{x}(k)} \quad \text { for all } x \in \Gamma, k \in N^{*} .
$$

Finally, applying Theorem 5.2 we conclude, for all $x, y \in \Gamma$ and $k \in N^{*}$,

$$
h_{2 k}(x, y) \leq \frac{C}{\sqrt{\tilde{f}_{x}(k) \tilde{f}_{y}(k)}} \exp \left(-\frac{d^{2}(x, y)}{D k}\right) .
$$

\section{REFERENCES}

[ 1 ] D. G. Aronson, Bounds for the fundamental solution of a parabolic equation, Bull. Amer. Math. Soc. 73 (1967), 890-896.

[2] P. Auscher AND T. Coulhon, Gaussian lower bounds for random walks from elliptic regularity, Ann. Inst. H. Poincaré Probab. Statist. 35 (1999), 605-630.

[3] P. Auscher, T. Coulhon And A. Grigor'YAn, ed., "Heat kernels and analysis on manifolds, graphs, and metric spaces (Paris, 2002)”, Contemp. Math. 338. Amer. Math Soc., Providence, R.I., 2003.

[ 4 ] M. T. BARlow, Random walks on supercritical percolation clusters, Ann. Probab. 32 (2004), 3024-3084.

[ 5 ] T .K. CARne, A transmutation formula for Markov chains, Bull. Sci. Math. (2) 109 (1985), 399-405.

[6] T. Coulhon, Analysis on infinite graphs with regular volume growth, Random walks and discrete potential theory (Cortona, 1997), 165-187, Sympos. Math. XXXIX, Cambridge Univ. Press, Cambridge, 1999.

[ 7 ] T. Coulhon, Random walks and geometry on infinite graphs, Lecture notes on analysis in metric spaces (Trento, 1999), 5-30, Appunti Corsi Tenuti Docenti Sc., Scuola Norm. Sup., Pisa, 2000.

[ 8 ] T. Coulhon And A. GRIGOR'Yan, On-diagonal lower bounds for heat kernels and Markov chains, Duke Math. J. 89 (1997), 133-199. 
[9] T. COUlhon AND A. GRIGOR'YAn, Random walks on graphs with regular volume growth, Geom. Funct. Anal. 8 (1998), 656-701.

[10] T. Coulhon And A. Grigor'yan, Pointwise estimates for transition probabilities of random walks on infinite graphs, Fractals in Graz 2001, 119-134, Trends Math., Birkäuser, Basel, 2003.

[11] T. Coulhon And L. Saloff-Coste, Puissances d'un opérateur régularisant, Ann. Inst. H. Poincaré Probab. Statist. 26 (1990), 419-436.

[12] E. B. DAVIES, Heat kernel bounds, conservation of probability and the Feller property, J. Anal. Math. 58 (1992), 99-119.

[13] T. Delmotte, Parabolic Harnack inequality and estimates of Markov chains on graphs, Rev. Mat. Iberoamericana 15 (1999), 181-232.

[14] A. GRIGOR'YAN, Heat kernel upper bounds on a complete non-compact manifold, Rev. Mat. Iberoamericana 10 (1994), 395-452.

[15] A. GRIGOR'YAN, Integral maximum principle and its applications, Proc. Roy. Soc. Edinburgh Sect. A 124 (1994), 353-362.

[16] A. GRIGOR'YAN, Gaussian upper bounds for the heat kernel on arbitrary manifolds, J. Differential Geom. 45 (1997), 33-52.

[17] A. GRIGOR'YAN, Estimates of heat kernels on Riemannian manifolds, Spectral theory and geometry, (Edinburgh 1998), 140-225, London Math. Soc. Lecture Note Ser. 273 Cambridge Univ. Press, Cambridge, 1999.

[18] W. Hebisch And L. SAloff-Coste, Gaussian estimates for Markov chains and random walks on groups, Ann. Probab. 21 (1993), 673-709.

[19] P. Li AND S.-T. YAU, On the parabolic kernel of the Schrödinger operator, Acta Math. 156 (1986), $153-201$.

[20] P. Mathieu And E. Remy, Décroissance du noyau de la chaleur et isopérimétrie sur un amas de percolation, C. R. Acad. Sci. Paris Sér. I Math. 332 (2001), 927-931.

[21] P. Mathieu And E. Remy, Isoperimetry and heat kernel decay on percolation clusters, Ann. Probab. 32 (2004), 100-128.

[22] Ch. Pittet And L. Saloff-Coste, A survey on the relationship between volume growth, isoperimetry, and the behavior of simple random walk on Cayley graphs, with examples, unpublished manuscript.

[23] D. W. Stroock, Estimates on the heat kernel for the second order divergence form operators, Probability theory (Singapore, 1989), 29-44, de Gruyter, Berlin, 1992.

[24] N. Th. VARopoulos, Long range estimates for Markov chains, Bull. Sci. Math. (2) 109 (1985), $225-252$.

[25] N. Th. Varopoulos, L. Saloff-Coste and T. Coulhon, Analysis and geometry on groups, Cambridge Tracts in Math. 100, Cambridge Univ. Press, Cambridge, 1992.

[26] W. WoEss, Random walks on infinite graphs and groups-a survey on selected topics, Bull. London Math. Soc. 26 (1994), 1-60.

Université DE CERGY-Pontoise 2

RUE ADOLPHE CHAUVIN

95302 PONTOISE

FRANCE

E-mail address: thierry.coulhon@math.u-cergy.fr
DEPARTMENT OF MATHEMATICS

IMPERIAL COLLEGE

LONDON SW7 2AZ

UNITED KINGDOM

E-mail address: a.grigoryan@ic.ac.uk

DiPARTIMENTO Di MATEMATICA

POLITECNICO Di Milano

PiAZZA LEONARDO DA VINCI 32

20133 MILANO

ITALY

E-mail address: zucca@mate.polimi.it 